ACTA MYCOLOGICA

Vol. 44 (2): 151-163

2009
Dedicated to Professor Krystyna Czyżewska

in honour of 40 years of her scientific activity

\title{
Epiphytic lichens of apple orchards in Poland, Slovakia, and Italy
}

\author{
DARIA ZARABSKA ${ }^{1}$, ANNA GUTTOVÁ ${ }^{2}$, FABIANA CRISTOFOLINI ${ }^{3}$, \\ PAOLO GIORDANI ${ }^{4}$ and ANNA LACKOVICOVÁ ${ }^{2}$
}

\footnotetext{
${ }^{1}$ Natural History Collection, Faculty of Biology, Adam Mickiewicz University, Umultowska 89 PL-61-614 Poznań, darzarabs12@tlen.pl

${ }^{2}$ Institute of Botany, Slovak Academy of Sciences, Dúbravská cesta 14, SK-845 23 Bratislava

${ }^{3}$ IASMA Research and Innovation Centre, Fondazione Edmund Mach-Environment and Natural Resources Area, via E. Mach, I-38010 S. Michele all'Adige (Trento)

${ }^{4}$ Botanic Centre Hanbury, University of Genova, Corso Dogali 1M, I-16146 Genova
}

Zarabska D., Guttová A., Cristofolini F., Giordani P., Lackovičová A.: Epiphytic lichens of apple orchards in Poland, Slovakia, and Italy. Acta Mycol. 44 (2): 151-163, 2009.

Following the study of epiphytic lichens in 30 apple orchards from Poland, Slovakia and Italy the list of 74 taxa was prepared. The most common are the meso- to xerophytic and heliophilous species. The highest number of taxa was observed in Slovak orchards. Moreover, lichens shared with at least one other country were also noted mainly in Slovakia. Bark of apple trees seems to create favourable habitats for Bacidia rubella, which together with Strangospora pinicola were valuable founds in Polish orchards. In Slovak orchards, special attention should be paid to Acrocordia gemmata, Melanelixia glabra and Usnea hirta. Among interesting records in Italian orchards, Phaeophyscia hispidula and Ph. kairamoi can be mentioned.

Key words: lichens, culture landscape, fruit trees, Wielkopolska province, Trentino region, Western Carpathians

\section{INTRODUCTION}

In accordance with the assumption of sustainable development policy, the conservation of old orchards can play an important role in supporting the wide variety of wildlife. The presence of old orchards in agricultural land can rise species diversity (cf. Jermaczek, Jermaczek 2003). Moreover, orchards as an example of culture landscape, can influence the spreading of some species (Szczepańska 2008).

Until now, some lichenological inventories were conducted in the orchards (e.g., Suza 1936a; Halicz, Kuziel 1958; Kuziel 1964; Kiss 1982; Mészáros et al. 1984; Czyżewska 1998; Bartók 1999; Kiszka 1999; Lipnicki 2003; Fałtynowicz et al. 2004; Kościelniak 2004; Lush et al. 2005; Lipnicki, Sobieralska 2009). Among flagship 
examples of research focused on the investigation of biota in the fruit trees should be distinguish studies carried in Czechia (Majeriková-Hlaváčková 1957), Great Britain (Lush et al. 2005), Hungary (Mészáros et al. 1984), Poland (Kuziel 1964; Lipnicki, Sobieralska 2009) and Romania (Bartók 1999). Records coming from studies of lichens only in apple orchards are scarce (Mészáros et al. 1984; Bartók 1999). More information is given from fruit trees level without direct reference to investigation of orchards (e.g., Endlicher 1830; Zahlbruckner 1894; Suza 1936a, b; Rydzak 1970; Toborowicz 1976; Lipnicki 1982; Kiszka, Piórecki 1991, 1994; Lackovičová 1997; Czyżewska 1998; Śliwa 1998; Lisická 1999; Czarnota 2000; Suppan, Mayrhofer 2002; Zalewska et al. 2004; Guttová 2005; Aptroot et al. 2006; John 2006; Pišút et al. 2007; Szymczyk, Zalewska 2008).

The aim of this research was to study the lichen species in a traditional apple orchards in the Wielkopolska province (western Poland), Western Carpathians (western and central Slovakia), and Trentino region (northern Italy). List of currently recorded species and a review of older data are treated in this paper.

\section{STUDY AREA}

Studied orchards in Poland were chosen on the border of Równina Nowotomyska plain and Wał Lwówecko-Rakoniewicki rampart being a part of Wielkopolska province (Kondracki 2000). Most of orchards investigated here were surrounded by crops and pasture land. Mainly they laid in the neighborhood of farms.

Region represent typical lowland character situated in the agricultural landscape. The altitude range creates on the level of 70-100 $\mathrm{m}$ a.s.l. The annual average temperature is $8.3^{\circ} \mathrm{C}$ and the average annual precipitation is ca $550 \mathrm{~mm}$ (averages from years 1971-2000; acc. to Farata 2004). The prevailing winds come from W, SW and NW.

Localities; 04-05. 2008: 1. Władysławowo village near Wąsowo, alt. $114 \mathrm{~m}, 52^{\circ} 23.44^{\prime} \mathrm{N}, 1^{\circ} 14.30^{\prime} \mathrm{E}$; 2. Wytomyśl-Lipka Mała village near Lwówek town, alt. 127 m, 52²3.37’ N, $16^{\circ} 11.07 ’ \mathrm{E}$; 3. Lipka Wielka village near Lwówek town, alt. 120 m, 52²4.03’N, $16^{\circ} 11.05^{\prime} \mathrm{E} ; 4$. Wytomyśl - Lipka Mała village near Lwówek town, alt. $127 \mathrm{~m}, 52^{\circ} 23.40^{\prime} \mathrm{N}, 16^{\circ} 11.10^{\prime} \mathrm{E} ; \mathbf{5}$. Wytomyśl village near Nowy Tomyśl town, alt. $101 \mathrm{~m}$, $52^{\circ} 23.0^{\prime} \mathrm{N}, 16^{\circ} 10.36^{\prime} \mathrm{E}$; 6. Kozie Laski village near Nowy Tomyśl town, alt. $96 \mathrm{~m}, 52^{\circ} 21.38^{\prime} \mathrm{N}, 16^{\circ} 10.15^{\prime} \mathrm{E}$; 7. Lipka Wielka village near Lwówek town, alt. $121 \mathrm{~m}, 52^{\circ} 24.17^{\prime} \mathrm{N}, 16^{\circ} 11.14$ 'E; 8. Wytomyśl village near Nowy Tomyśl town, alt. 105 m, 52²2.37’N, 16²10.58’E; 9. Wytomyśl village near Nowy Tomyśl town, alt. $106 \mathrm{~m}, 52^{\circ} 22.37^{\prime} \mathrm{N}, 16^{\circ} 10.58^{\prime} \mathrm{E} ; \mathbf{1 0}$. Wytomyśl village near Nowy Tomyśl town, alt. $107 \mathrm{~m}, 52^{\circ} 22.35^{\prime} \mathrm{N}$, $16^{\circ} 10.33^{\prime} \mathrm{E}$.

All Slovak orchards were localized in Western Carpathians Mts., namely in Malé Karpaty Mts., Biele Karpaty Mts., Štiavnické vrchy Mts., Nízke Tatry Mts., and Stolické vrchy Mts. The sites are situated from 160 m (Bratislava, Malé Karpaty Mts.) to $730 \mathrm{~m}$ a.s.l. (Hybe, Nízke Tatry Mts.). Orchards were located in cadasters of the villages (city), in the vicinity of farms/housing estates, or were surrounded by pasture land and in the neighborhood of forest. The Western Carpathians are characterized by mountain climate with clearly marked vertical zonation (Bielczyk et al. 2004). Annual precipitations range from 600 to $800-1200 \mathrm{~mm}$. 
Localities; 06.2008: 1. Biele Karpaty Mts., Poriadie-Durcovci village near Stará Turá town, alt. 380 m, 48 $46.34^{\prime} \mathrm{N}, 17^{\circ} 37.33^{\prime} \mathrm{E}$; 2. Malé Karpaty Mts., Bratislava, beside the road to Záhorská Bystrica village, alt. $160 \mathrm{~m}, 48^{\circ} 13.11^{\prime} \mathrm{N}, 17^{\circ} 02.02^{\prime} \mathrm{E} ; 3$. Biele Karpaty Mts., Adamovské Kochanovce village, locality Vinohrady, alt. 250 m, 48 $52.23^{\prime} \mathrm{N}, 17^{\circ} 54.59^{\prime} \mathrm{E}$; 4. Biele Karpaty Mts., Adamovské Kochanovce village, alt. $220 \mathrm{~m}, 48^{\circ} 51.09^{\prime} \mathrm{N}, 17^{\circ} 55.05^{\prime} \mathrm{E}$; 5. Nízke Tatry Mts., Hybe, near the saw mill in N part of village, alt. $730 \mathrm{~m}, 49^{\circ} 03.15^{\prime} \mathrm{N}, 19^{\circ} 50.10^{\prime} \mathrm{E} ;{ }^{\circ}$. Stolické vrchy Mts., Revúca, NW part of the town, ca $500 \mathrm{~m}$ from the railway, alt. $320 \mathrm{~m}, 48^{\circ} 41.20^{\prime} \mathrm{N}, 20^{\circ} 06.30^{\prime} \mathrm{E}$; 7. Biele Karpaty Mts., Trenčín-Orechové, N part of the village by Orechový potok stream, alt. $350 \mathrm{~m}, 48^{\circ} 54.2^{\prime} \mathrm{N}, 18^{\circ} 02.15^{\prime} \mathrm{E} ; 8$. Biele Karpaty Mts., Nová Bošáca village, Grúň Mt., alt. $550 \mathrm{~m}, 48^{\circ} 53.43^{\prime} \mathrm{N}, 17^{\circ} 47.42^{\prime} \mathrm{E}$; 9. Štiavnické vrchy Mts., Pukanec village, alt. 354 $\mathrm{m}, 48^{\circ} 22.08^{\prime} \mathrm{N}, 18^{\circ} 44.02^{\prime} \mathrm{E} ; 10$. Štiavnické vrchy Mts., Pukanec-Majere, locality Teplá voda, alt. $370 \mathrm{~m}$, $48^{\circ} 22.16^{\prime} \mathrm{N}, 18^{\circ} 45.02^{\prime} \mathrm{E}$.

Italian apple orchards were selected in the Non Valley (Trentino region - N Italy). The Non Valley takes shape in western Trentino, along the shores of the Noce Stream from the Rocchetta ravin up to the Palade and Mendola Pass, between 500 and $1400 \mathrm{~m}$ a.s.l. The valley is characterized by an intensive agricultural landscape, with a strong prevalence of apple orchards, which produce up to $15 \%$ of the Italian production. The climate is Alpine, with average yearly precipitation between 500 and $1200 \mathrm{~mm}$ and average yearly temperature about $9^{\circ} \mathrm{C}$.

Localities; 03-04.2009: 1. Near the Santa Giustina Lake, alt. $530 \mathrm{~m}, 46^{\circ} 21.52^{\prime} \mathrm{N}, 11^{\circ} 03.30^{\prime} \mathrm{E} ;$ 2. Near $^{\circ}$ the Santa Giustina Lake, along the road, alt. $540 \mathrm{~m}, 46^{\circ} 21.52^{\prime} \mathrm{N}, 11^{\circ} 03.27^{\prime} \mathrm{E}$; 3. Near the Santa Giustina Lake, alt. $595 \mathrm{~m}, 46^{\circ} 20.45^{\prime} \mathrm{N}, 11^{\circ} 03.23^{\prime} \mathrm{E}$; 4. Near Sarnonico, alt. $970 \mathrm{~m}, 46^{\circ} 25.17^{\prime} \mathrm{N}, 11^{\circ} 08.34^{\prime} \mathrm{E}$; 5. Sarnonico, along the road to Seio, alt. $970 \mathrm{~m}, 46^{\circ} 25.17^{\prime} \mathrm{N}, 11^{\circ} 08.34^{\prime} \mathrm{E}$; 6. Sarnonico, along the road to Seio, alt. $970 \mathrm{~m}, 46^{\circ} 25.11^{\prime} \mathrm{N}, 11^{\circ} 07.58^{\prime} \mathrm{E}$; 7. Sarnonico, along the road to Seio, alt. $960 \mathrm{~m}, 46^{\circ} 25.11^{\prime} \mathrm{N}, 11^{\circ} 07.55^{\prime} \mathrm{E}$; 8 . Sarnonico, along the road to Cavareno, alt. $1000 \mathrm{~m}, 46^{\circ} 24.44^{\prime} \mathrm{N}, 11^{\circ} 08.29^{\prime} \mathrm{E}$; 9. Near the Santa Giustina Lake, along the road to Sanzeno, alt. $590 \mathrm{~m}, 46^{\circ} 21.45^{\prime} \mathrm{N}, 11^{\circ} 03.50^{\prime} \mathrm{E}$; 10. Near the Santa Giustina Lake, along the road to Sanzeno, alt. $580 \mathrm{~m}, 46^{\circ} 21.42^{\prime} \mathrm{N}, 11^{\circ} 03.42^{\prime} \mathrm{E}$.

\section{MATERIAL AND METHODS}

In every orchard one sample plot $(20 \times 50 \mathrm{~m})$ in direction north-south was established. From each stand lichens were recorded carefully from the bark of apple (Malus sp.) up to $1.6 \mathrm{~m}$. Nomenclature of lichens follows Nimis and Martellos (2008). Voucher specimen are available at the $\mathrm{POZ}$ herbarium.

\section{RESULTS AND DISCUSSION}

In total 74 taxa were recorded in the investigated orchards of three countries (Tab.1). Similar results (in species number) were obtained by Kościelniak (2004). Specific circumstances prevailing in the orchards can have impact on occurrence of some species. Apple orchards in comparison with other fruit plantations are characterized by quite good light conditions and difficult access to the water on the trunk (Kuziel 1964). Moreover, favorable conditions for some lichens can be also created by bark $\mathrm{pH}$ of Malus sp. which is naturally higher in comparison with some other trees, e.g., 


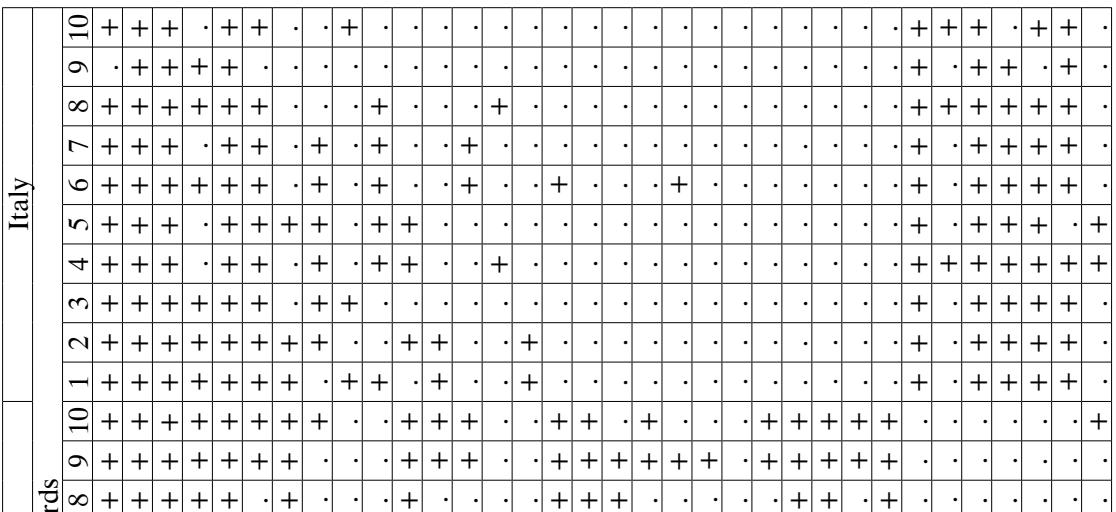

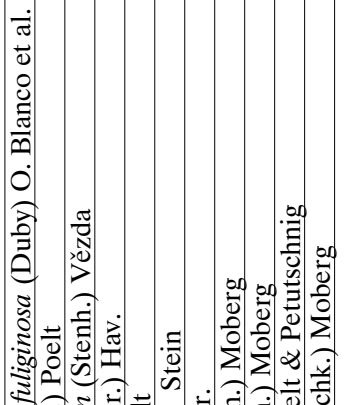

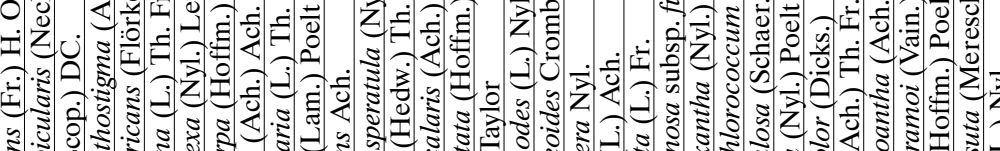

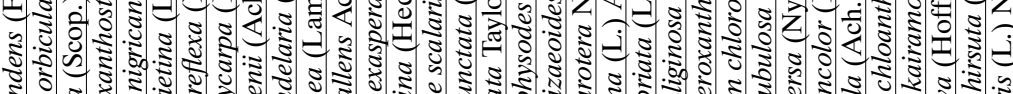

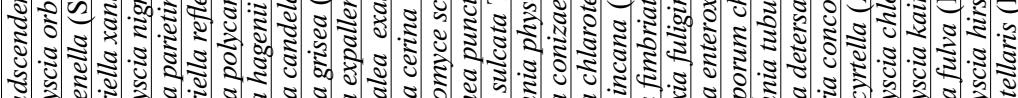

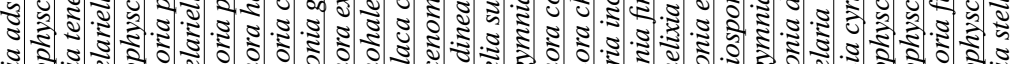

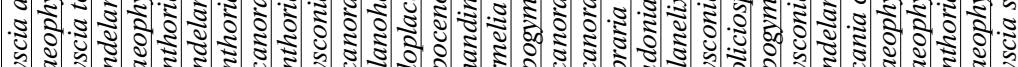

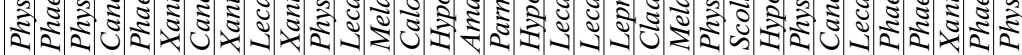




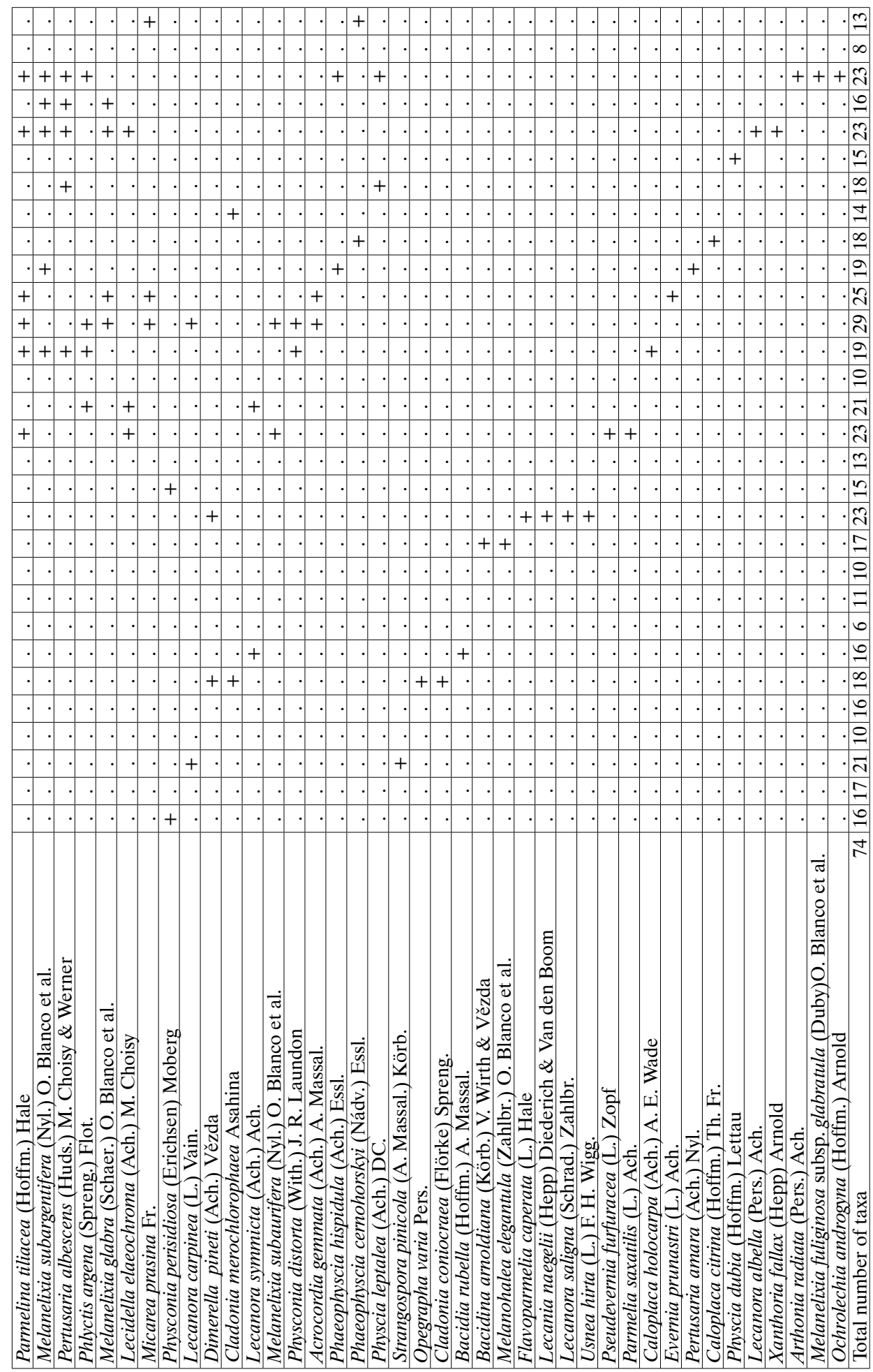


oak (Svoboda 2007), and can promote occurrence of some specific, like nitrophytic, group of lichens. Most of recorded in each country lichens have high indicator value for light (Wirth 2001), e.g., Caloplaca cerina, Candelariella xanthostigma, Cladonia fimbriata, Hypogymnia physodes, Lecanora conizaeoides, Melanohalea exasperatula, Parmelia sulcata, Phaeophyscia nigricans, Ph. orbicularis, Physcia adscendens, Ph. tenella, Physconia enteroxantha, Ph. grisea, Xanthoria candelaria, X. parietina and $X$. polycarpa. Some species - Candelariella xanthostigma, Phaeophyscia nigricans, Ph. orbicularis, Physcia adscendens, Ph. tenella, Xanthoria parietina and X. polycarpa represent mesophytic or xerophytic character (Nimis, Martellos 2008).

Most richest in species were orchards in Slovakia (52). Higher species richness can be partially explained by wider altitudinal range (Dietrich, Scheidegger 1997). Less records were made in Polish (32) and Italian (43) orchards. Climatic conditions, especially rainfall (Lush et al. 2005) is one of the main factor impacted diversity of observed lichens in the particular countries. In region of Poland where studies were conducted one of the smallest amount of rainfall (Farata 2004) in comparison with other parties of Poland as well as with studied sites in Slovakia and Italy can be observed. Such climatic conditions surely negatively influenced growing of lichens. Most of foliose broad-lichens represent montane elements in the achieved list of orchards lichens. Some of narrow-lobed lichens (e.g., Phaeophyscia chloantha and $P h$. hirsuta), recorded in alpine region of Trentino represent submediterranean element in the lichen biota. Nevertheless such species like Phaeophyscia kairamoi, $P h$. hispidula and Ph. cernohorskyi often noted in Italian orchards are common and abundant in Alpine regions, and in this situation impact of elevation on their notes in the orchards could play the role.

Despite records of some rare lichens for particular countries (e.g., Bacidia rubella, Melanelixia glabra, Physconia perisidiosa and Usnea hirta) most of identified species are widespread. Such situation seems to characterized non-forested rural areas (Giordani 2006), in which also orchards can be distinguished. Species diversity dwindle to rather simplified flora due to also agricultural (Loppi, de Dominicis 1996). On the other hand records of some species included to the national/regional red lists confirm some observations about importance of anthropogenic habitats for occurrence of some valuable lichens (e.g., Lush et al. 2005).

Lichenbiota of Slovakia and Poland is much close to each other in comparison with results obtained from Italy. It can be explained by more similar climatic conditions of both countries connected with their geographical localization. On the other hand similarity of lichens records in Slovak and Italian orchards can be connected with altitudinal influence on the distribution of some species. Both in Slovakia and Italy orchards were situated mainly or only in mountains regions where specific conditions could favorable some common species for both countries.

Most of recently noted lichens were observed during earlier studies conducted on epiphytes of fruit trees (Tab. 2). Mainly ubiquitous species with a broad geographical range were found in most of the investigated orchards: Candelariella xanthostigma, Phaeophyscia orbicularis, Ph. nigricans, Physcia adscendens, Ph. tenella, Xanthoria parietina, and $X$. polycarpa. All of above mentioned taxa are mesophytic or xerophytic and grow in rather sun-exposed sites (Nimis, Martellos 2008).

Considering previous data the species recorded mainly at apple orchards are: Caloplaca cerina, Lecania cyrtella, and Xanthoria candelaria. Especially Caloplaca 
Table 2

Bibliography of lichens recorded during the present study on Malus sp., both solitary and in orchards, in Poland, Slovakia, and Italy

\begin{tabular}{|c|c|c|}
\hline Species & Bark of Malus sp. solitary & Bark of Malus sp. in orchard \\
\hline $\begin{array}{l}\text { Acrocordia } \\
\text { gemmata }\end{array}$ & $\begin{array}{l}\text { Kiszka, Piórecki 1991, 1994; Suppan, } \\
\text { Mayrhofer } 2002\end{array}$ & Kościelniak 2004 \\
\hline $\begin{array}{l}\text { Amandinea } \\
\text { punctata }\end{array}$ & $\begin{array}{l}\text { Rydzak 1970; Toborowicz 1976; Lipnicki } \\
\text { 1982; Czyżewska 1998; Czarnota 2000; } \\
\text { Suppan, Mayrhofer 2002; Guttová 2005; } \\
\text { John 2006; Szymczyk, Zalewska } 2008\end{array}$ & $\begin{array}{l}\text { Bartók 1999; Kiszka 1999; } \\
\text { Kościelniak 2004; Lush et al. 2005; } \\
\text { Lipnicki, Sobieralska } 2009\end{array}$ \\
\hline Arthonia radiata & - & $\begin{array}{l}\text { Mészáros et al. 1984; Bartók 1999; } \\
\text { Lush et al. } 2005\end{array}$ \\
\hline Bacidia rubella & $\begin{array}{l}\text { Kiszka, Piórecki 1991; Śliwa 1998; Czarnota } \\
\text { 2000; Suppan, Mayrhofer 2002; Guttová } \\
\text { 2005 }\end{array}$ & $\begin{array}{l}\text { Suza 1936a; Kuziel 1964; Bartók } \\
\text { 1999; Kiszka 1999; Kościelniak } \\
\text { 2004; Lush et al. 2005 }\end{array}$ \\
\hline Caloplaca cerina & Rydzak 1970; Suppan, Mayrhofer 2002 & $\begin{array}{l}\text { Suza 1936a; Kuziel 1964; Mészáros } \\
\text { et al. 1984; Bartók 1999 }\end{array}$ \\
\hline Caloplaca citrina & - & Kuziel 1964 \\
\hline $\begin{array}{l}\text { Caloplaca } \\
\text { holocarpa }\end{array}$ & - & $\begin{array}{l}\text { Bartók 1999; Lipnicki, Sobieralska } \\
2009\end{array}$ \\
\hline $\begin{array}{l}\text { Candelaria } \\
\text { concolor }\end{array}$ & $\begin{array}{l}\text { Rydzak 1970; Toborowicz 1976; Kiszka, } \\
\text { Pírecki 1991, 1994; Sliwa 1998; Czarnota } \\
2000\end{array}$ & $\begin{array}{l}\text { Kuziel 1964; Mészáros et al. } \\
\text { 1984; Bartók 1999; Kiszka 1999; } \\
\text { Kościelniak 2004; Lush et al. } 2005\end{array}$ \\
\hline $\begin{array}{l}\text { Candelariella } \\
\text { reflexa }\end{array}$ & $\begin{array}{l}\text { Śliwa 1998; Lisická 1999; Suppan, Mayrhofer } \\
\text { 2002; Guttová 2005, Szymczyk, Zalewska } \\
\text { 2008 }\end{array}$ & $\begin{array}{l}\text { Kiszka, Piórecki 1991; Czarnota } \\
\text { 2000; Lush et al. 2005 }\end{array}$ \\
\hline $\begin{array}{l}\text { Candelariella } \\
\text { xanthostigma }\end{array}$ & $\begin{array}{l}\text { Rydzak 1970; Lackovičová 1978; Kiszka, } \\
\text { Piórecki 1991; Lisická 1997; Czarnota } \\
\text { 2000; Zalewska et al. 2004; Guttová 2005; } \\
\text { Szymczyk, Zalewska 2008 }\end{array}$ & $\begin{array}{l}\text { Kuziel 1964; Toborowicz 1976; } \\
\text { Czyżewska 1998; Kiszka 1999; } \\
\text { Kościelniak 2004; Pišút } 2008 \\
\text { (unpubl.); Lipnicki, Sobieralska } \\
\text { 2009 }\end{array}$ \\
\hline $\begin{array}{l}\text { Cladonia } \\
\text { coniocraea }\end{array}$ & Suppan, Mayrhofer 2002 & $\begin{array}{l}\text { Kościelniak 2004; Lush et al. 2005; } \\
\text { Lipnicki, Sobieralska } 2009\end{array}$ \\
\hline Cladonia fimbriata & $\begin{array}{l}\text { Kiszka, Piórecki 1991, 1994; Suppan, } \\
\text { Mayrhofer 2002; Szymczyk, Zalewska } 2008\end{array}$ & $\begin{array}{l}\text { Kościelniak 2004; Lush et al. 2005; } \\
\text { Lipnicki, Sobieralska } 2009\end{array}$ \\
\hline Dimerella pineti & Kiszka, Piórecki 1991 & Kościelniak 2004 \\
\hline Evernia prunastri & $\begin{array}{l}\text { Endlicher 1830; Zahlbruckner 1894; } \\
\text { Majeriková-Hlaváčková 1957; Rydzak 1970; } \\
\text { Toborowicz 1976; Kiszka, Piórecki 1991, } \\
\text { 1994; Suppan, Mayrhofer 2002; Szymczyk, } \\
\text { Zalewska 2008 }\end{array}$ & $\begin{array}{l}\text { Kuziel 1964; Lipnicki 2003; } \\
\text { Kościelniak 2004; Lush et al. 2005; } \\
\text { Lipnicki, Sobieralska } 2009\end{array}$ \\
\hline $\begin{array}{l}\text { Flavoparmelia } \\
\text { caperata }\end{array}$ & $\begin{array}{l}\text { Zahlbruckner 1894; Rydzak 1970; Kiszka, } \\
\text { Piórecki 1991, 1994; Czarnota 2000; Guttová } \\
\text { 2005; John 2006 }\end{array}$ & $\begin{array}{l}\text { Kuziel 1964; Bartók 1999; } \\
\text { Kościelniak 2004; Lush et al. } 2005\end{array}$ \\
\hline $\begin{array}{l}\text { Hypocenomyce } \\
\text { scalaris }\end{array}$ & Lipnicki 1982 & $\begin{array}{l}\text { Kościelniak 2004; Lipnicki, } \\
\text { Sobieralska 2009 }\end{array}$ \\
\hline $\begin{array}{l}\text { Hypogymnia } \\
\text { physodes }\end{array}$ & $\begin{array}{l}\text { Majeriková-Hlaváčková 1957; Halicz, } \\
\text { Kuziel 1958; Rydzak 1970, Toborowicz 1976; } \\
\text { Lackovičová 1978; Lipnicki 1982; Kiszka, } \\
\text { Piórecki 1991, 1994; Czarnota 2000; Suppan, } \\
\text { Mayrhofer 2002; Zalewska et al. 2004; John } \\
\text { 2006; Szymczyk, Zalewska } 2008\end{array}$ & $\begin{array}{l}\text { Kuziel 1964; Bartók 1999; Kiszka } \\
\text { 1999; Kościelniak 2004; Lush et } \\
\text { al. 2005; Pišút 2008 (unpubl.); } \\
\text { Lipnicki, Sobieralska 2009 }\end{array}$ \\
\hline $\begin{array}{l}\text { Hypogymnia } \\
\text { tubulosa }\end{array}$ & $\begin{array}{l}\text { Lisická 1999; Czarnota 2000; Suppan, } \\
\text { Mayrhofer 2002; John } 2006\end{array}$ & $\begin{array}{l}\text { Kuziel 1964; Kościelniak 2004; } \\
\text { Lush et al. 2005; Lipnicki, } \\
\text { Sobieralska 2009 }\end{array}$ \\
\hline Lecania cyrtella & - & $\begin{array}{l}\text { Kuziel 1964; Mészáros et al. 1984; } \\
\text { Czyżewska 1998; Bartók 1999; } \\
\text { Kościelniak 2004 }\end{array}$ \\
\hline Lecania naegelii & Suppan, Mayrhofer 2002 & $\begin{array}{l}\text { Kościelniak 2004; Kuziel 1964; } \\
\text { Lush et al. } 2005\end{array}$ \\
\hline
\end{tabular}


Table 2 cont.

\begin{tabular}{|c|c|c|}
\hline Lecanora albella & - & Lush et al. 2005 \\
\hline Lecanora carpinea & $\begin{array}{l}\text { Rydzak 1970; Toborowicz 1976; Lackovičová } \\
\text { 1978; Kiszka, Piórecki 1991, 1994; Suppan, } \\
\text { Mayrhofer 2002; Aptroot et al. 2006 }\end{array}$ & $\begin{array}{l}\text { Halicz, Kuziel 1958; Kuziel 1964; } \\
\text { Mészáros et al. 1984; Bartók } \\
\text { 1999; Kościelniak 2004; Lipnicki, } \\
\text { Sobieralska 2009 }\end{array}$ \\
\hline $\begin{array}{l}\text { Lecanora } \\
\text { chlarotera }\end{array}$ & $\begin{array}{l}\text { Rydzak 1970; Lisická 1997; Suppan, } \\
\text { Mayrhofer 2002; Aptroot et al. 2006; } \\
\text { Szymczyk, Zalewska } 2008\end{array}$ & $\begin{array}{l}\text { Kuziel 1964; Kościelniak 2004; } \\
\text { Lush et al. } 2005\end{array}$ \\
\hline $\begin{array}{l}\text { Lecanora } \\
\text { conizaeoides }\end{array}$ & Lackovičová 1978; Lipnicki 1982 & $\begin{array}{l}\text { Bartók 1999; Kiszka 1999; } \\
\text { Kościelniak 2004; Lipnicki, } \\
\text { Sobieralska 2009 }\end{array}$ \\
\hline Lecanora expallens & $\begin{array}{l}\text { Rydzak 1970; Toborowicz 1976; Lipnicki } \\
\text { et al. 1991; Kiszka, Piórecki 1991, 1994; } \\
\text { Zalewska et al. 2004; John 2006 }\end{array}$ & $\begin{array}{l}\text { Kiszka 1999; Kościelniak 2004; } \\
\text { Lipnicki, Sobieralska } 2009\end{array}$ \\
\hline Lecanora hagenii & Rydzak 1970; Lisická 1997 & Kościelniak 2004 \\
\hline Lecanora saligna & Śliwa 1998; Czarnota 2000 & $\begin{array}{l}\text { Czyżewska 1998; Kościelniak 2004; } \\
\text { Lipnicki, Sobieralska } 2009\end{array}$ \\
\hline $\begin{array}{l}\text { Lecanora } \\
\text { symmicta }\end{array}$ & John 2006 & Bartók 1999; Kościelniak 2004 \\
\hline $\begin{array}{l}\text { Lecidella } \\
\text { elaeochroma }\end{array}$ & $\begin{array}{l}\text { Rydzak 1970; Lipnicki 1982; Kiszka, Piórecki } \\
\text { 1991, 1994; Suppan, Mayrhofer 2002; } \\
\text { Guttová 2005; Aptroot et al. 2006; Szymczyk, } \\
\text { Zalewska 2008 }\end{array}$ & $\begin{array}{l}\text { Kuziel 1964; Bartók 1999; Kiszka } \\
\text { 1999; Kościelniak 2004; Lush et al. } \\
\text { 2005; Lipnicki, Sobieralska } 2009\end{array}$ \\
\hline Lepraria incana & $\begin{array}{l}\text { Kiszka, Piórecki 1991, 1994; Zalewska et al. } \\
\text { 2004, John } 2006\end{array}$ & $\begin{array}{l}\text { Bartók 1999; Kiszka 1999; } \\
\text { Kościelniak 2004; Lipnicki, } \\
\text { Sobieralska 2009 }\end{array}$ \\
\hline $\begin{array}{l}\text { Melanohalea } \\
\text { elegantula }\end{array}$ & Suppan, Mayrhofer 2002 & Kuziel 1964; Kościelniak 2004 \\
\hline $\begin{array}{l}\text { Melanohalea } \\
\text { exasperatula }\end{array}$ & $\begin{array}{l}\text { Rydzak 1970; Kiszka, Piórecki 1991, 1994; } \\
\text { Czyżewska 1998; Czarnota 2000; Suppan, } \\
\text { Mayrhofer 2002; Zalewska et al. 2004, John } \\
\text { 2006; Szymczyk, Zalewska } 2008 \\
\end{array}$ & $\begin{array}{l}\text { Suza 1936a; Kuziel 1964; } \\
\text { Toborowicz 1976; Kościelniak 2004; } \\
\text { Lipnicki, Sobieralska } 2009\end{array}$ \\
\hline $\begin{array}{l}\text { Melanelixia } \\
\text { fuliginosa }\end{array}$ & $\begin{array}{l}\text { Majeriková-Hlaváčková 1957; Rydzak 1970; } \\
\text { Kiszka, Piórecki 1991, 1994; Czarnota 2000; } \\
\text { Suppan, Mayrhofer 2002; Zalewska et al. } \\
\text { 2004; Guttová 2005; John 2006; Lisická et } \\
\text { al. 2008 }\end{array}$ & $\begin{array}{l}\text { Halicz, Kuziel 1958; Kuziel 1964; } \\
\text { Kościelniak 2004; Lush et al. } 2005\end{array}$ \\
\hline Melanelixia glabra & $\begin{array}{l}\text { Zahlbruckner 1894; Lisická 1997; Lisická et } \\
\text { al. } 2008\end{array}$ & $\begin{array}{l}\text { Suza 1936a; Kuziel 1964; } \\
\text { Kościelniak } 2004\end{array}$ \\
\hline $\begin{array}{l}\text { Melanelixia } \\
\text { subargentifera }\end{array}$ & $\begin{array}{l}\text { Zahlbruckner 1894, 1905; Kiszka, Piórecki } \\
\text { 1991; Lisická } 1997\end{array}$ & Kuziel 1964; Kościelniak 2004 \\
\hline $\begin{array}{l}\text { Melanelixia } \\
\text { subaurifera }\end{array}$ & $\begin{array}{l}\text { Rydzak 1970; Kiszka, Piórecki 1991, 1994; } \\
\text { Suppan, Mayrhofer } 2002\end{array}$ & $\begin{array}{l}\text { Kuziel 1964; Kościelniak 2004; } \\
\text { Lush et al. } 2005\end{array}$ \\
\hline Opegrapha varia & Rydzak 1970 & $\begin{array}{l}\text { Bartók 1999; Lipnicki, Sobieralska } \\
2009\end{array}$ \\
\hline Parmelia saxatilis & $\begin{array}{l}\text { Majeriková-Hlaváčková 1957; Halicz, } \\
\text { Kuziel 1958; Rydzak 1970; Toborowicz } \\
\text { 1976; Lipnicki 1982; Kiszka, Piórecki 1991, } \\
\text { 1994; Piśút, Lackovičová 1993; Lisická 1997; } \\
\text { Czyżewska 1998; Czarnota 2000; Suppan, } \\
\text { Mayrhofer 2002; Zalewska et al. 2004; John } \\
\text { 2006; Szymczyk, Zalewska 2008 }\end{array}$ & $\begin{array}{l}\text { Kuziel 1964; Mészáros et al. } \\
\text { 1984; Kiszka 1999; Bartók 1999; } \\
\text { Kościelniak 2004; Lush et al. 2005; } \\
\text { Pišút } 2008 \text { (unpubl.) }\end{array}$ \\
\hline Parmelina tiliacea & $\begin{array}{l}\text { Zahlbruckner 1894; Kiszka, Piórecki } \\
\text { 1991, 1994; Aptroot et al. 2006; Szymczyk, } \\
\text { Zalewska 2008 }\end{array}$ & Bartók 1999; Kościelniak 2004 \\
\hline $\begin{array}{l}\text { Pertusaria } \\
\text { albescens }\end{array}$ & $\begin{array}{l}\text { Śliwa 1998; Czarnota 2000; Suppan, } \\
\text { Mayrhofer 2002; Guttová 2005; John } 2006\end{array}$ & Kościelniak 2004 \\
\hline Pertusaria amara & $\begin{array}{l}\text { Kiszka, Piórecki 1991, 1994; Suppan, } \\
\text { Mayrhofer } 2002\end{array}$ & $\begin{array}{l}\text { Mészáros et al. 1984; Bartók 1999; } \\
\text { Kościelniak 2004; Lush et al. } 2005\end{array}$ \\
\hline $\begin{array}{l}\text { Phaophyscia } \\
\text { chloantha }\end{array}$ & Kiszka, Piórecki 1991 & Kościelniak 2004 \\
\hline
\end{tabular}


Table 2 cont.

\begin{tabular}{|c|c|c|}
\hline $\begin{array}{l}\text { Phaeophyscia } \\
\text { hirsuta }\end{array}$ & $\begin{array}{l}\text { Kiszka, Piórecki 1991, 1994; Lisická 1999; } \\
\text { Lisická et al. } 2008\end{array}$ & Kościelniak 2004 \\
\hline $\begin{array}{l}\text { Phaeophyscia } \\
\text { nigricans }\end{array}$ & $\begin{array}{l}\text { Rydzak 1970; Toborowicz 1976; Kiszka, } \\
\text { Piórecki 1991, } 1994\end{array}$ & $\begin{array}{l}\text { Kuziel 1964; Kiszka 1999; Lipnicki, } \\
\text { Sobieralska } 2009\end{array}$ \\
\hline $\begin{array}{l}\text { Phaeophyscia } \\
\text { orbicularis }\end{array}$ & $\begin{array}{l}\text { Rydzak 1970; Kiszka, Piórecki 1991, 1994; } \\
\text { Suppan, Mayrhofer 2002; Guttová 2005; } \\
\text { Lisická et al. 2008; Szymczyk, Zalewska } 2008\end{array}$ & $\begin{array}{l}\text { Kuziel 1964; Mészáros et al. } \\
\text { 1984; Bartók 1999; Kiszka 1999; } \\
\text { Kościelniak 2004; Lush et al. 2005; } \\
\text { Pišút } 2008 \text { (unpubl.); Lipnicki, } \\
\text { Sobieralska } 2009\end{array}$ \\
\hline Phlyctis argena & $\begin{array}{l}\text { Rydzak 1970; Toborowicz 1976; Lipnicki } \\
\text { 1982; Kiszka, Piórecki 1991, 1994; Czarnota } \\
\text { 2000; Suppan, Mayrhofer 2002; Zalewska et } \\
\text { al. 2004; John } 2006\end{array}$ & $\begin{array}{l}\text { Kiszka 1999; Kościelniak 2004; } \\
\text { Lush et al. 2005; Lipnicki, } \\
\text { Sobieralska } 2009\end{array}$ \\
\hline $\begin{array}{l}\text { Physcia } \\
\text { adscendens }\end{array}$ & $\begin{array}{l}\text { Majeriková-Hlaváčková 1957; Rydzak } \\
\text { 1970; Toborowicz 1976; Lackovičová 1978; } \\
\text { Lipnicki 1982; Kiszka, Piórecki 1991, 1994; } \\
\text { Czarnota 2000; John 2006; Aptroot et al. } \\
\text { 2006; Szymczyk, Zalewska 2008 }\end{array}$ & $\begin{array}{l}\text { Mészáros et al. 1984; Bartók 1999; } \\
\text { Kiszka 1999; Kościelniak 2004; } \\
\text { Pišút } 2008 \text { (unpubl.); Lipnicki, } \\
\text { Sobieralska } 2009\end{array}$ \\
\hline Physcia dubia & $\begin{array}{l}\text { Rydzak 1970; Toborowicz 1976, Szymczyk, } \\
\text { Zalewska } 2008\end{array}$ & $\begin{array}{l}\text { Kuziel 1964; Lipnicki 2003; } \\
\text { Kościelniak 2004; Lipnicki, } \\
\text { Sobieralska } 2009\end{array}$ \\
\hline Physcia stellaris & $\begin{array}{l}\text { Zahlbruckner 1894; Rydzak 1970; } \\
\text { Lackovičová 1978; Kiszka, Piórecki 1991; } \\
\text { Lisická 1997; Szymczyk, Zalewska 2008 }\end{array}$ & $\begin{array}{l}\text { Kuziel 1964; Kiszka 1999; } \\
\text { Kościelniak } 2004\end{array}$ \\
\hline Physcia tenella & $\begin{array}{l}\text { Rydzak 1970; Toborowicz 1976; Lipnicki } \\
\text { 1982; Kiszka, Piórecki 1991, 1994; Czarnota } \\
\text { 2000; Suppan, Mayrhofer 2002; Zalewska } \\
\text { et al. 2004; John 2006; Lisická et al. 2008; } \\
\text { Szymczyk, Zalewska 2008 }\end{array}$ & $\begin{array}{l}\text { Suza 1936a; Kuziel 1964; } \\
\text { Czyżewska 1998; Bartók 1999; } \\
\text { Kiszka 1999; Kościelniak 2004; } \\
\text { Lush et al. 2005; Lipnicki, } \\
\text { Sobieralska 2009 }\end{array}$ \\
\hline Physconia detersa & $\begin{array}{l}\text { Majeriková-Hlaváčková 1957; Rydzak 1970; } \\
\text { Toborowicz } 1976\end{array}$ & Kościelniak 2004 \\
\hline Physconia distorta & $\begin{array}{l}\text { Zahlbruckner 1894; Kiszka, Piórecki 1991, } \\
\text { 1994; Czarnota 2000; Suppan, Mayrhofer } \\
\text { 2002; John } 2006\end{array}$ & $\begin{array}{l}\text { Kościelniak 2004; Lipnicki, } \\
\text { Sobieralska } 2009\end{array}$ \\
\hline $\begin{array}{l}\text { Physconia } \\
\text { enteroxantha }\end{array}$ & $\begin{array}{l}\text { Kiszka, Piórecki 1991, 1994; Czarnota 2000; } \\
\text { John 2006, Szymczyk, Zalewska } 2008\end{array}$ & $\begin{array}{l}\text { Kościelniak 2004; Lipnicki, } \\
\text { Sobieralska 2009 }\end{array}$ \\
\hline Physconia grisea & $\begin{array}{l}\text { Suza 1936b; Rydzak 1970; Toborowicz 1976; } \\
\text { Lisická 1997; John 2006; Lisická et al. } 2008\end{array}$ & $\begin{array}{l}\text { Kuziel 1964; Bartók 1999; Kiszka } \\
\text { 1999; Kościelniak 2004; Lipnicki, } \\
\text { Sobieralska } 2009\end{array}$ \\
\hline $\begin{array}{l}\text { Physconia } \\
\text { perisidiosa }\end{array}$ & Lisická 1997; Czarnota 2000; John 2006 & $\begin{array}{l}\text { Lipnicki 2003; Kościelniak 2004; } \\
\text { Lipnicki, Sobieralska } 2009\end{array}$ \\
\hline $\begin{array}{l}\text { Pseudevernia } \\
\text { furfuracea }\end{array}$ & $\begin{array}{l}\text { Majeriková-Hlaváčková 1957; Toborowicz } \\
\text { 1976; Kiszka, Piórecki 1991, 1994; Lisická } \\
\text { 1999; Aptroot et al. 2006; John 2006 } \\
\end{array}$ & Kuziel 1964; Kościelniak 2004 \\
\hline $\begin{array}{l}\text { Scoliciosporum } \\
\text { chlorococcum }\end{array}$ & $\begin{array}{l}\text { Toborowicz 1976; Kiszka, Piórecki 1991, } \\
\text { 1994; Czarnota 2000; Suppan, Mayrhofer } \\
2002\end{array}$ & $\begin{array}{l}\text { Kiszka 1999; Kościelniak 2004; } \\
\text { Lipnicki, Sobieralska } 2009\end{array}$ \\
\hline Usnea hirta & Rydzak 1970; Zahlbruckner 1894 & Kuziel 1964 \\
\hline $\begin{array}{l}\text { Xanthoria } \\
\text { candelaria }\end{array}$ & Rydzak 1970; Toborowicz 1976; Śliwa 1998 & $\begin{array}{l}\text { Kuziel 1964; Mészáros et al. } \\
\text { 1984; Bartók 1999; Kiszka 1999; } \\
\text { Kościelniak 2004; Lipnicki, } \\
\text { Sobieralska 2009 }\end{array}$ \\
\hline Xanthoria fallax & $\begin{array}{l}\text { Rydzak 1970; Kiszka, Piórecki 1991, 1994; } \\
\text { Czarnota } 2000\end{array}$ & $\begin{array}{l}\text { Kuziel 1964; Kiszka 1999; } \\
\text { Kościelniak } 2004\end{array}$ \\
\hline $\begin{array}{l}\text { Xanthoria } \\
\text { parietina }\end{array}$ & $\begin{array}{l}\text { Szatala 1916; Majeriková-Hlaváčková } \\
\text { 1957; Rydzak 1970; Lackovičová 1978; } \\
\text { Lipnicki 1982; Kiszka, Piórecki 1991, 1994; } \\
\text { Czyżewska 1998; Lisická 1999; Czarnota } \\
\text { 2000; Szymczyk, Zalewska 2008 }\end{array}$ & $\begin{array}{l}\text { Kuziel 1964; Toborowicz 1976; } \\
\text { Bartók 1999; Kiszka 1999; } \\
\text { Kościelniak 2004; Lush et al. 2005; } \\
\text { Pišút 2008 (unpubl.); Lipnicki, } \\
\text { Sobieralska 2009 }\end{array}$ \\
\hline $\begin{array}{l}\text { Xanthoria } \\
\text { polycarpa }\end{array}$ & $\begin{array}{l}\text { Rydzak 1970; Kiszka, Piórecki 1991,1994; } \\
\text { Aptroot et al. 2006; John 2006; Szymczyk, } \\
\text { Zalewska 2008 }\end{array}$ & $\begin{array}{l}\text { Kuziel 1964; Toborowicz 1976; } \\
\text { Kościelniak 2004; Lush et al. 2005; } \\
\text { Lipnicki, Sobieralska } 2009\end{array}$ \\
\hline
\end{tabular}


cerina should be paid attention; Wirth (2002) treats this species as an indicator of orchards. Notes about Bacidina arnoldiana, Cladonia merochlorophaea, Micarea prasina, Ochrolechia androgyna, Phaeophyscia cernohorskyi, Ph. hispidula, Ph. kairamoi, Physcia leptalea, Strangospora pinicola, and Xanthoria fulva are not given from Malus sp. solitary and apple orchards in cited literature.

Among recorded species of special interest Bacidia rubella should be distinguished. The species was recorded only once in one of the richest in lichens orchards in Poland. Mentioned above lichen was found associated with: Candelariella reflexa, Melanohalea exasperatula, Parmelia sulcata, Phaeophyscia nigricans, Ph. orbicularis, and Physcia adscendens. Considering "Red list of the lichens in Poland" (Cieśliński et al. 2006) Bacidia rubella is included to the group of species under special attention. Bark of apple trees seems to be one of the main substrate for Bacidia rubella. The species was recorded only (Suza 1936a; Kuziel 1964; Śliwa 1998) or mainly on this phorophyte (Kiszka 1999; Suppan, Mayrhofer 2002; Kościelniak 2004; Guttová 2005). Wirth (1995) considers apple bark in the orchards as an important habitats for its occurrence. Among other species recorded in the Polish orchards a record of Strangospora pinicola is interesting. The species was observed only in one locality of all investigated orchards. Previously no data about its occurrence in this kind of biotope were known. In Poland Strangospora pinicola is described with the status least concern (Cieśliński et al. 2006). Sun exposed sites on which species mainly occur (Nimis, Martellos 2008) can be found in the apple orchards (Kuziel 1964) and probably it was main factor positively influenced on growing of Strangospora pinicola in this ecosystem.

In Slovak orchards records of Acrocordia gemmata, Melanelixia glabra (VU) and Usnea hirta (VU) should be highlighted. Acrocordia gemmata occurs mostly in natural and semi-natural woods (e.g., Lisická et al. 2008), the data from the past were scarse (Pišút 1999). The second species occurs in the areas with higher precipitation, in open sites, mostly in semi-natural woods tolerated low degree of eutrophication (Wirth 1995). Although reported from relatively many localities in the Malé Karpaty Mts. (Lackovičová 1978) and also during mapping of epiphytic lichens in Slovakia in 1970-1981 (Pišút 1999), any further report is valuable. Usnea hirta is one of the most frequently collected small species of the genus in Slovakia after changes of air quality followed by transformation of industry after 1989. Juvenile thalli can be found in urban areas occurring within nitrophilous Xanthorion communities (e.g., Nimis 1981; Fos, Clerc 2000) or in former areas influenced by industrial emissions.

Among commonly recorded lichens in Italian orchards species from genus Phaeophyscia should be distinguished. Ph. hispidula and Ph. kairamoi should be paid special attention. Both of these taxa are restricted to the northern part of Italy considering their distribution pattern in this country (Nimis, Martellos 2008). From Italy $P h$. hispidula is reported as being at least very rare in particular regions of it's occurrence (Trentino-Alto Adige, Lombardia). This species can be found on very acid to subneutral substrate in sites with very weak to weak eutrophication. Notes of $P h$. kairamoi come from Trentino-Alto Adige and Friuli and it's probably restricted to Italian Alps. It's heliophilous species occurring in sun-exposed sites were recorded on base-rich bark (Nimis, Martellos 2008). 


\section{CONCLUSIONS}

Dissimilarity between number and recorded species in the studied sites can be the consequence of differences in genus and number of available phorophytes. In apple orchards the same bark and similar number of investigated trees (connected with restricted area of plots) minimizes this problem. It was the reason why so many common species were found between particular orchards and countries. Orchards seems to create their own specific conditions which favors some groups of organisms, e.g., nitrophilous, heliophilous. Nevertheless, occurrence of some rare species should pointed necessity of focusing more attention on this less studied type of agroecosystem.

Acknowledgements. DZ acknowledges the Ministry of Education of the Slovak Republic, Slovenská Akademická Informačná Agentúra (SAIA, APVV-51-040805, VEGA 0071), and Ministry of Foreign Affairs in Italy (3306 dell'11/09/2008) for financially support. Authors are grateful Dr. Ivan Pišút for use of his unpublished data from orchards, and anonymous reviewer for useful suggestions that helped improved the manuscript.

\section{REFERENCES}

Aptroot A., John V., Wirth V. 2006. Flechten und lichenicole Pilze im Dreiländereck ber der Saarschleife mit Neufunden aus Lothringen, Saarland und Rheinland-Pfalz (BLAM-Exkursion 2005). Herzogia 19: $63-76$.

Bartók K. 1999. Pesticide usage and epiphytic lichen diversity in Romanian orchards. Lichenologist 31 (1): $21-25$.

Bielczyk U., Lackovičová A., Farkas E.E., Lökös L., Liška J., Breuss O., Kondratyuk S. Ya. 2004. Checklist of lichens of the Western Carpathians. Biodiversity of the Carpathians 1: 1-181. W. Szafer Institute of Botany, Polish Academy of Sciences, Kraków.

Cieśliński S., Czyżewska K., Fabiszewska J. 2006. Red list of the lichens in Poland. (In:) Z. Mirek, K. Zarzycki, W. Wojewoda, Z. Szeląg (eds). Red list of plants and fungi in Poland. 3. ed.: 71-89. W. Szafer Institute of Botany, Polish Academy of Sciences, Kraków.

Czarnota P. 2000. Porosty Gorczańskiego Parku Narodowego. Część I. Wykaz i rozmieszczenie gatunków. Parki nar. Rez. Przyr. 19 (1): 3-73.

Czyżewska K. 1998. Lichenized Ascomycotina of the Bełchatów Industrial Region (Central Poland). Acta Mycol. 33 (2): 341-366.

Dietrich M., Scheidegger C. 1997. Frequency, diversity and ecological strategies of epiphytic lichens in the Swiss Central Plateau and the Pre-Alps. Lichenologist 29 (3): 237-258.

Endlicher S. 1830. Flora posoniensis, exhibens plantas circa Posonium sponte crescentes aut frequentius cultas, methodo naturali dispositas. Posonii.

Fałtynowicz W., Krzysztofiak A., Krzysztofiak L., Zalewska A., Świerubska T. 2004. Porosty. Rutka-Rutka $1.2 \mathrm{~km}$. Ścieżka poznawcza. Stowarzyszenie „Człowiek i Przyroda”, Suwałki.

Farata R. (ed.). 2004. Atlas klimatu województwa wielkopolskiego. IMGW, Poznań.

Fos S., Clerc P. 2000. The lichen genus Usnea on Quercus suber in Iberia cork-oak forests. Lichenologist 32: $67-88$.

Giordani P. 2006. Variables influencing distribution of epiphytic lichens in heterogeneous area: A case study for Liguria, NW Italy. Journal of Vegetation Science 17: 195-206.

Guttová A. 2005. Charakteristika diverzity lišajníkov slovenskej časti Pienin a pril’ahlého územia. Biosozologia 3: 23-45.

Halicz B., Kuziel S. 1958. Materiały do flory porostów Wyżyny Łódzkiej. Zesz. Nauk. UŁ 2, Nauki Mat.Przyr. 4: 101-125.

Jermaczek A., Jermaczek M. 2003. Ocalmy stare sady. Wydawnictwo Klubu Przyrodników, Świebodzin.

John V. 2006. Flechten und flechtenbewohnende Pilze auf dem Nackberg im Saarland (Ergebnisse vom Tag der Artenvielfalt 2006). Abh. DELATTINIA 32: 113-125. 
Kiss T. 1982. Áttekintés és adatok a gyümölcsfák zuzmóflórájáról és a fajok indikátor szerepéről. Bot. Közlem. 68: 1-17.

Kiszka J. 1999. Porosty (Lichenes) oraz warunki bioekologiczne Przemyśla. Arbor. Bolestr. 6: 3-86. Bolestraszyce.

Kiszka J., Piórecki J. 1991. Porosty Lichenes Pogórza Przemyskiego. UNIWA, Warszawa.

Kiszka J., Piórecki J. 1994. Waloryzacja przyrodnicza porostów (Lichenes) projektowanego Turnickiego Parku Narodowego. (In:) J. Kiszka, J. Piórecki (eds). Porosty apofityczne jako wynik antropopresji. Arbor. Bolestr. 2: 95-122. Bolestraszyce.

Kondracki J. 2000. Geografia regionalna Polski. Państwowe Wydawnictwo Naukowe, Warszawa.

Kościelniak R. 2004. Porosty (Lichenes) Bieszczadów Niskich. Fragm. Florist. Geobot. Polonica, Suppl. 5: $1-164$.

Kuziel S. 1964. Porosty epifityczne drzew owocowych w kotlinie Łącka i okolicy. Łódzkie Tow. Nauk., Prace Wydz. III Nauk Mat.-Przyr. 98: 1-42.

Lackovičová A. 1978. Lišajníky Malých Karpát. Acta Ecol. 6 (1977), 15: 7-107.

Lackovičová A. 1997. Aktuálny výskyt lišajníkov v Národnej prirodnej rezervácii Šúr. Bull. Slov. Bot. Spoločn. 19: 71-77.

Lipnicki L. 1982 (1984). Porosty miasta Drezdenka i najbliższej okolicy. Fragm. Florist. Geobot. 28 (2): 221-239.

Lipnicki L. 2003. Porosty nadrzewne w starych sadach przydomowych. (In:) R. Sobieralska (ed.). Poradnik sadowniczy starych odmian drzew owocowych. TPDW, Świecie: 77-82.

Lipnicki L., Sobieralska R. 2009. Porosty epifityczne w starych sadach w Dolinie Dolnej Wisły. Parki nar. Rez. Przyr. 28 (1): 37-49.

Lisická E. 1997. Lišajníky (Lichenes). (In:) V. Feráková (ed.). V. Flóra, geológia a paleontológia Devínskej Kobyly. APOP, Bratislava: 72-77.

Lisická E. 1999. Prispevok k poznaniu lišajnikov Vel'kej Fatry. Acta Rer. natur. Mus. Nat. Slov. 45: $7-16$.

Lisická E., Pišút I., Kliment J. 2008. Lišajníky. (In:) J. Kliment (ed.). Príroda Vel'kej Fatry. Lišajníky, machorasty, cievnaté rastliny. Vydavatel'stvo UK, Bratislava: 33-62.

Loppi S., de Dominicis V. 1996. Effects of agriculture on epiphytic lichen vegetation in central Italy. Israel Journal of Plant Sciences 44: 297-307.

Lush M., Robertson H. J., Alexander K. N. A., Giavarini V., Hewins E., Mellings J., Stevenson C. R., Storey M. 2005. The extent, distribution, biodiversity and management of traditional orchards in England. Case Studies. English Nature, Peterborough.

Majeriková-Hlaváčková J. 1957. Lišejníky ovocných stromů. Rostlinna Výroba 3 (30): 141-156.

Mészáros Z., Ádám L., Balázs K., Benedek I., Csikai Cs., Draskovits Á.D. Kozár F., Lővei G., Mahunka S., Meszleny A., Mihályi F., Mihályi K., Nagy L., Oláh B., Papp J., Papp L., Polgár L., Radwan Z., Rácz V., Ronkay L., Solymosi P., Soós A., Szabóky S., Szabóky Cs., Szalay-Marzsó L., Szarukán I., Szelényi G., Szentkirályi F., Sziráki Gy., Szöke L., Török, J. 1984. Results of Faunistical and Floristical Studies in Hungarian Apple Orchards (Apple Ecosystem Research No. 26). Acta Phytopathologica Academiae Scientiarum Hungaricae 19: 91-176.

Nimis P. L. 1981. Epiphytic lichen vegetation in the Lumiei-Valley (Carnian Alps). Gortania 3: 123-142.

Nimis P. L., Martellos S. 2008. ITALIC - The Information System on Italian Lichens. Version 4.0. University of Trieste, Dept. of Biology, IN4.0/1 (http://dbiodbs.univ.trieste.it/).

Pišút I. 1999. Mapovanie rozšírenia epifytických lišajníkov na Slovensku (1970-1981). Botanický ústav SAV, Bratislava.

Pišút I., Lackovičová A. 1993. Lišajníky. (In:) L. Bertová (ed.). Karlova Ves, Vlastivedná monografia. Alfa, Bratislava: 36-38.

Pišút I., Lackovičová A., Guttová A., Palice Z. 2007. New lichen records from Bukovské vrchy Mts. (NE Slovakia). Acta Mycol. 42 (2): 267-280.

Rydzak J. 1970. Flora i ekologia porostów drzew przydrożnych. Ann. UMCS, C 25 (15): 149-157.

Suppan U., Mayrhofer H. 2002. Beiträge zur Flechtenflora von Slovenien VI. Die epiphytischen Flechten der Uršlja gora. Herzogia 15: 57-77.

Suza J. 1936a. Lišejniky Piešt'anska. Carpatica 1: 275-331.

Suza J. 1936b. Lišejníky Velké Fatry (Slovensko). Sborn. Muz. Slov. Spoločn. 30: 111-129.

Svoboda D. 2007. Evaluation of the European method for mapping lichen diversity (LDV) as an indicator of environmental stress in the Czech Republic. Biologia 62 (4): 424-431. 
Szatala Ö. 1916. Adatok Ung vármegye zuzmóflórájának ismeretéhez. Bot. Közlem. 15: 1-34.

Szczepańska K. 2008. Antropogeniczne przemiany bioty porostów Masywu Śnieżnika i Gór Bialskich. Acta Bot. Siles., Monogr. 4: 1-291.

Szymczyk R., Zalewska A. 2008. Lichens in the rural landscape of the Warmia Plain. Acta Mycol. 43 (2): $215-230$.

Śliwa L. 1998. Antropogeniczne przemiany lichenoflory Beskidu Sądeckiego. Prace Bot. 31, 158 pp.

Toborowicz K. 1976. Porosty miasta Kielc i najbliższej okolicy. Fragm. Florist. Geobot. 22 (4): 575-603.

Wirth V. 1995. Die Flechten Baden-Württembergs. Ulmer, Stuttgart.

Wirth V. 2001. Zeigerwerte von Flechte. 3. Aufl. Scripta Geobotanica 18: 221-243.

Wirth V. 2002. Indicator Flechte. Naturschutz aus der Flechten-Perspektive. Stuttgarter Beiträge zur Naturkunde. Serie C - Wiessen für alle. Heft 50, Sttutgart.

Zahlbruckner A. 1894. Zur Flechtenflora des Pressburger Komitates. Verh. Vereins Natur-Heilk., Pressburg, Neue Folge 8 (1892/93): 19-73, 273-284.

Zahlbruckner A. 1905. Neue Beiträge zur Flechtenflora des Pozsonyer Komitates. Verh. Vereins NaturHeilk. Pressburg, Neue Folge 16 (1904): 119-131.

Zalewska A., Fałtynowicz W., Krzysztofiak A., Krzysztofiak L., Picińska-Fałtynowicz J. 2004. Lichens of Suwalski Landscape Park. (In:) A. Zalewska, W. Fałtynowicz (eds). Lichens of the protected areas in the Euregion Niemen. "Man and Nature" Association, Suwałki: 5-50.

\section{Porosty epifityczne sadów jabłoniowych w Polsce, Słowacji i Włoszech}

\section{Streszczenie}

Przeprowadzone badania porostów epifitycznych w trzydziestu sadach w Polsce (10), Słowacji (10) i Włoszech (10) pozwoliły opracować listę 74 odnotowanych taksonów. Najbardziej rozpowszechnione gatunki należą do grupy taksonów mezo- i kserofitycznych oraz światłolubnych. W słowackich sadach zaobserwowano najwyższą liczbę gatunków oraz najwyższą liczbę gatunków wspólnych z przynajmniej jednym krajem. Wydaje się, że kora jabłoni stwarza dogodne warunki dla rozwoju Bacidia rubella, która razem ze Strangospora pinicola były cennymi porostami odnotowanymi w polskich sadach. W sadach badanych na terenie Słowacji szczególną uwagę zwróciły stanowiska Acrocordia gemmata, Melanelixia glabra i Usnea hirta. Phaeophyscia hispidula i Ph. kairamoi to interesujące porosty zaobserwowane w sadach włoskich. 